\title{
Tumor-associated macrophages promote ovarian cancer cell migration by secreting transforming growth factor beta induced (TGFBl) and tenascin C
}

Anna Mary Steitz', Alina Steffes², Florian Finkernagel', Annika Unger ${ }^{1}$, Leah Sommerfeld', Julia M. Jansen', Uwe Wagner ${ }^{3}$, Johannes Graumann (1) ${ }^{4,5}$, Rolf Müller (1) ${ }^{1}$ and Silke Reinartz ${ }^{2}$

\begin{abstract}
A central and unique aspect of high-grade serous ovarian carcinoma (HGSC) is the extensive transcoelomic spreading of tumor cell via the peritoneal fluid or malignant ascites. We and others identified tumor-associated macrophages (TAM) in the ascites as promoters of metastasis-associated processes like extracellular matrix (ECM) remodeling, tumor cell migration, adhesion, and invasion. The precise mechanisms and mediators involved in these functions of TAM are, however, largely unknown. We observed that HGSC migration is promoted by soluble mediators from ascites-derived TAM, which can be emulated by conditioned medium from monocyte-derived macrophages (MDM) differentiated in ascites to TAM-like asc-MDM. A similar effect was observed with IL-10-induced alternatively activated m2c-MDM but not with LPS/IFNy-induced inflammatory m1-MDM. These observations provided the basis for deconvolution of the complex TAM secretome by performing comparative secretome analysis of matched triplets of different MDM phenotypes with different pro-migratory properties (asc-MDM, m2c-MDM, m1-MDM). Mass spectrometric analysis identified an overlapping set of nine proteins secreted by both asc-MDM and m2c-MDM, but not by m1-MDM. Of these, three proteins, i.e., transforming growth factor beta-induced (TGFBI) protein, tenascin C (TNC), and fibronectin (FN1), have been associated with migration-related functions. Intriguingly, increased ascites concentrations of TGFBI, TNC, and fibronectin were associated with short progression-free survival. Furthermore, transcriptome and secretome analyses point to TAM as major producers of these proteins, further supporting an essential role for TAM in promoting HGSC progression. Consistent with this hypothesis, we were able to demonstrate that the migration-inducing potential of asc-MDM and m2c-MDM secretomes is inhibited, at least partially, by neutralizing antibodies against TGFBI and TNC or siRNA-mediated silencing of TGFBI expression. In conclusion, the present study provides the first experimental evidence that TAM-derived TGFBI and TNC in ascites promote HGSC progression.
\end{abstract}

\section{Introduction}

Ovarian carcinoma is the most lethal gynecological cancer with an overall 12 -year survival rate of $<20 \%$, and represents the fifth leading cause of cancer-associated deaths in females ${ }^{1}$. A hallmark of high-grade serous

\footnotetext{
Correspondence: Rolf Müller (rolf.mueller@uni-marburg.de)

${ }^{1}$ Institute of Molecular Biology and Tumor Research (IMT), Center for Tumor

Biology and Immunology, Philipps University, Marburg, Germany

${ }^{2}$ Clinic for Gynecology, Gynecologic Oncology and Endocrinology, Center for

Tumor Biology and Immunology, Philipps University, Marburg, Germany

Full list of author information is available at the end of the article

Edited by H.-U. Simon
}

carcinoma (HGSC), the most common and aggressive subtype, is its extensive peritoneal metastasis, which occurs at a very early stage of disease and contributes to its fatal clinical outcome ${ }^{2}$. Metastatic spreads occurs predominantly to the omentum and serous membranes lining the peritoneal organs through transcoelomic dissemination of tumor cells via the peritoneal fluid ${ }^{3-5}$. The peritoneal tumor microenvironment (TME), which consists of tumor-infiltrated host tissues and peritoneal fluid (or ascites at advanced stages), is an essential determinant of metastatic disease progression. Ascites contains large

\section{(c) The Author(s) 2020}

(c) (i) Open Access This article is licensed under a Creative Commons Attribution 4.0 International License, which permits use, sharing, adaptation, distribution and reproduction cc) in any medium or format, as long as you give appropriate credit to the original author(s) and the source, provide a link to the Creative Commons license, and indicate if changes were made. The images or other third party material in this article are included in the article's Creative Commons license, unless indicated otherwise in a credit line to the material. If material is not included in the article's Creative Commons license and your intended use is not permitted by statutory regulation or exceeds the permitted use, you will need to obtain permission directly from the copyright holder. To view a copy of this license, visit http://creativecommons.org/licenses/by/4.0/. 
numbers of cells including tumor spheroids and immune cells, such as tumor-associated macrophages and $\mathrm{T}$ cells (TAMs and TATs, respectively) ${ }^{6-8}$, as well as soluble factors and extracellular vesicles released by tumor and host cells ${ }^{2,9,10}$, collectively referred to as the tumor secretome and recognized as a key player in the communication network of the $\mathrm{TME}^{11-13}$. A detailed understanding of the secretome composition, the origin of single compounds, and their role in tumor-host crosstalk remains, however, elusive.

TAMs constitute a prominent cell population in ascites known to promote tumor growth, metastasis, and immunosuppression ${ }^{14-16}$. TAMs are reprogrammed by factors of the TME to adopt a pro-tumorigenic and immunosuppressive phenotype, which is linked to a poor clinical outcome $e^{8,13,17,18}$. TAMs contribute to the tumor secretome by releasing a plethora of soluble mediators, such as interleukin (IL)-6, IL-10, C-C chemokine motif ligand 18 (CCL18), CCL22, tumor necrosis factor $\alpha$, and transforming growth factor beta (TGF $\beta$ ), that trigger protumorigenic signaling pathways in both tumor and host cells of the $\mathrm{TME}^{19-21}$. For example, it is suggested that TAM and tumor cells cooperate in extracellular matrix (ECM) remodeling, which is a prerequisite for tumor cell adhesion and invasion ${ }^{7,13,14}$. Consistent with this model, it has been reported that TAM secrete migration-promoting factors like insulin-like growth factor 1 (IGF1), epidermal growth factor (EGF), and CHI3L1 pointing to a presumably central role of TAM in cancer cell migration, adhesion, and invasion ${ }^{22-24}$.

The TAM-derived mediators that promote cancer migration in the context of the HGSC microenvironment remain largely unknown. This is largely due to the fact that macrophages secrete a plethora of soluble factors, thus complicating the identification of relevant mediators. To address this issue, we designed an experimental setting that compares the secretomes of macrophages with different migration-stimulating properties. This was achieved by comparing matched pairs of monocytederived macrophages (MDM) from healthy donors that were differentiated into TAM-like MDM by ascites (ascMDM), alternatively activated M2 by IL-10 (m2c$\mathrm{MDM}^{25}$ as well as MDM classically activated by lipopolysaccharide (LPS) and interferon- $\gamma$ (IFN $\gamma$ ) (m1-MDM). While asc-MDM and m2c-MDM share the potential to stimulate HGSC cell migration similarly to patientderived TAMs, m1-MDM has no migration-promoting potential.

By combining mass spectrometry (MS)-based proteomics, bioinformatic analyses, and tumor migration assays, we found three candidates with migrationpromoting properties released by both asc-MDM and m2c-MDM, but not by m1-MDM. These secreted proteins were transforming growth factor beta induced
(TGFBI), tenascin C (TNC), and fibronectin (FN1), which have in common that they are ECM proteins and as such may provide support for tumor cell adhesion and migration. In general, excessive synthesis and deposition of ECM proteins is a hallmark of the tumor stroma, which is especially mediated not only by carcinoma-associated fibroblasts $(\mathrm{CAFs})^{26}$ but also by $\mathrm{TAM}^{13,27}$. So far, TNC and TGFBI secretion by TAM has not been linked to tumor cell migration. In the present study, we identified TGFBI, TNC, and FN1 in ascites and found correlations with HGSC progression, supporting a potential clinical relevance of these mediators in the TME. For TGFBI and TNC in particular, we provide evidence for enhanced secretion into the TME as a novel mechanism by which TAM promote HGSC migration.

\section{Results}

\section{Ascites-derived TAM secrete soluble mediators promoting HGSC migration}

As shown in Fig. 1, the secretome of ascites-derived TAM induced strong migration of cultured patientderived HGSC cells (termed OCMI cells) when applied as chemoattractant in a transwell assay. These findings were validated using conditioned media of TAM from three patients and tumor cells from five patients, indicating that ascites-derived TAM secrete migrationpromoting mediators acting on different OCMI tumor cells (Fig. 1a, b).

The highly complex composition of the TAM secretome, which consists of several hundred proteins, complicates the identification of the pro-migratory mediators that are relevant within the HGSC microenvironment. We therefore designed an experimental approach suitable to selectively identify the TAM-derived mediators able to promote HGSC cell migration (see also "Introduction"). This approach is based on our observation that MDM differentiated in vitro with malignant ascites into TAMlike MDM (asc-MDM) emulated the pro-migratory potential of the patients' TAM secretomes. This was demonstrated in a transwell migration assays, where conditioned medium from asc-MDM significantly induced tumor cell migration when used as chemoattractant (Fig. 1c and Supplementary Fig. S1). A similar impact on migration was observed by conditioned medium from MDM alternatively activated by IL-10 (m2cMDM), whereas migration was not significantly affected by conditioned medium MDM skewed to a proinflammatory phenotype by LPS and IFN $\gamma$ (m1-MDM) (Fig. 1d and Supplementary Fig. S1). These observations were confirmed in a second migration assay format, where conditioned media was used for pre-incubating tumor cells (prior to the migration assay) rather than as chemoattractant. As shown in Fig. 1e, tumor cells that were pre-incubated with conditioned media of asc-MDM and 


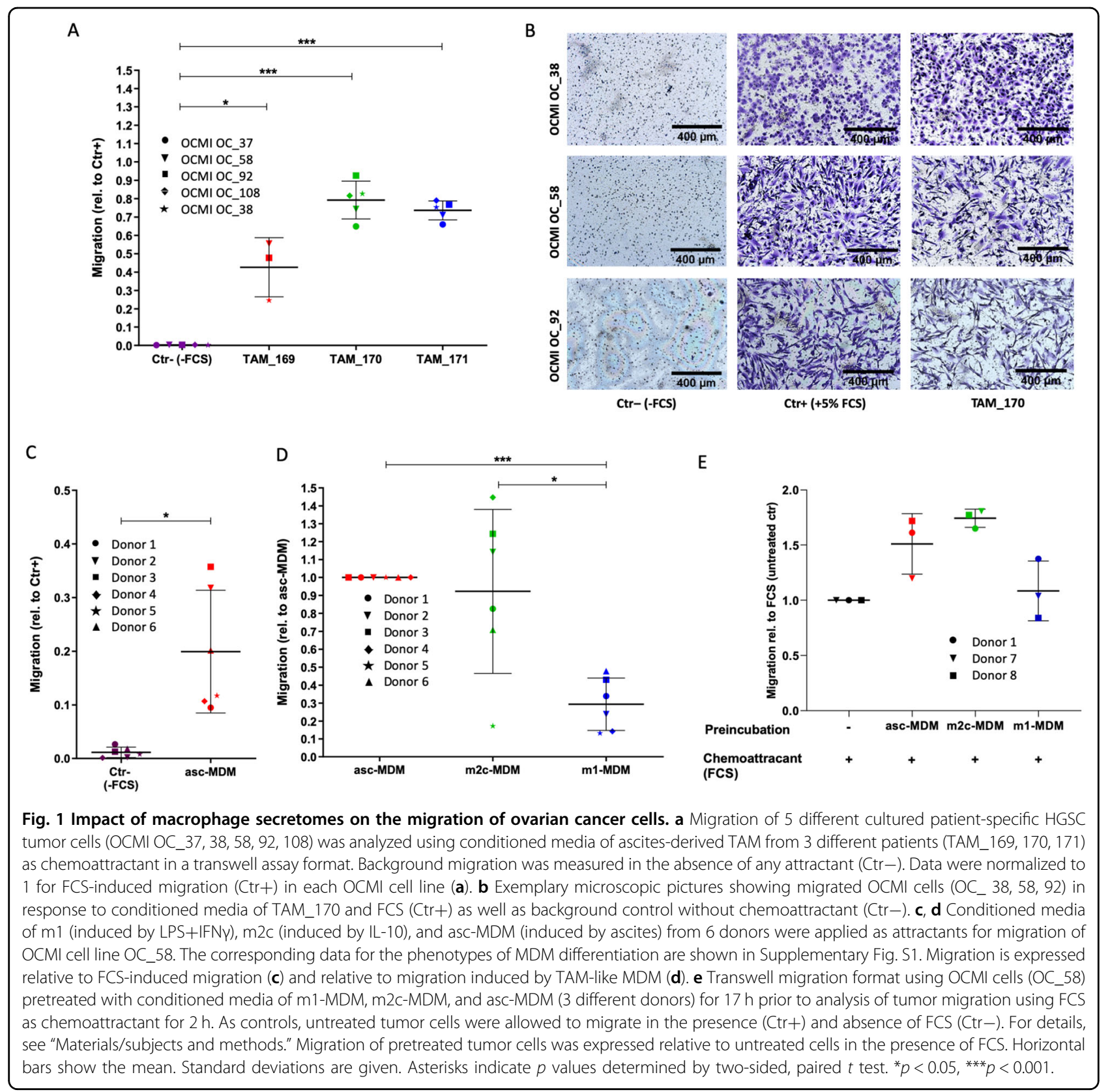

m2c-MDM, but not of m1-MDM, exhibited increased migratory potential (Fig. 1e).

The MDM differentiation phenotypes were also verified by flow cytometry (see Supplementary Fig. S2). TAM-like asc-MDM were characterized by an increased expression of CD14, CD16, and the m2c markers CD163 and CD206, as well as downregulated expression of the M1 markers CD86 and CCR7 in all tested donors (relative to m1MDM; Fig. S2).

The differential effects of asc-MDM and m2c-MDM on the one hand and m1-MDM on the other hand paved the way for a detailed comparative analysis of the corresponding secretomes aiming at the identification of candidate proteins with a pro-migratory function secreted by asc-MDM and m2c-MDM (and by TAM in malignant ascites) but not by m1-MDM.

\section{Comparative secretome analysis of MDM subtypes identifies candidates related to tumor migration}

Comparative analysis of conditioned media from functionally different MDM subtypes was performed by MSbased proteomics. In total, we identified 700 proteins annotated as "predicted secreted" in the Human Protein Atlas in the supernatant of at least one asc-MDM sample 

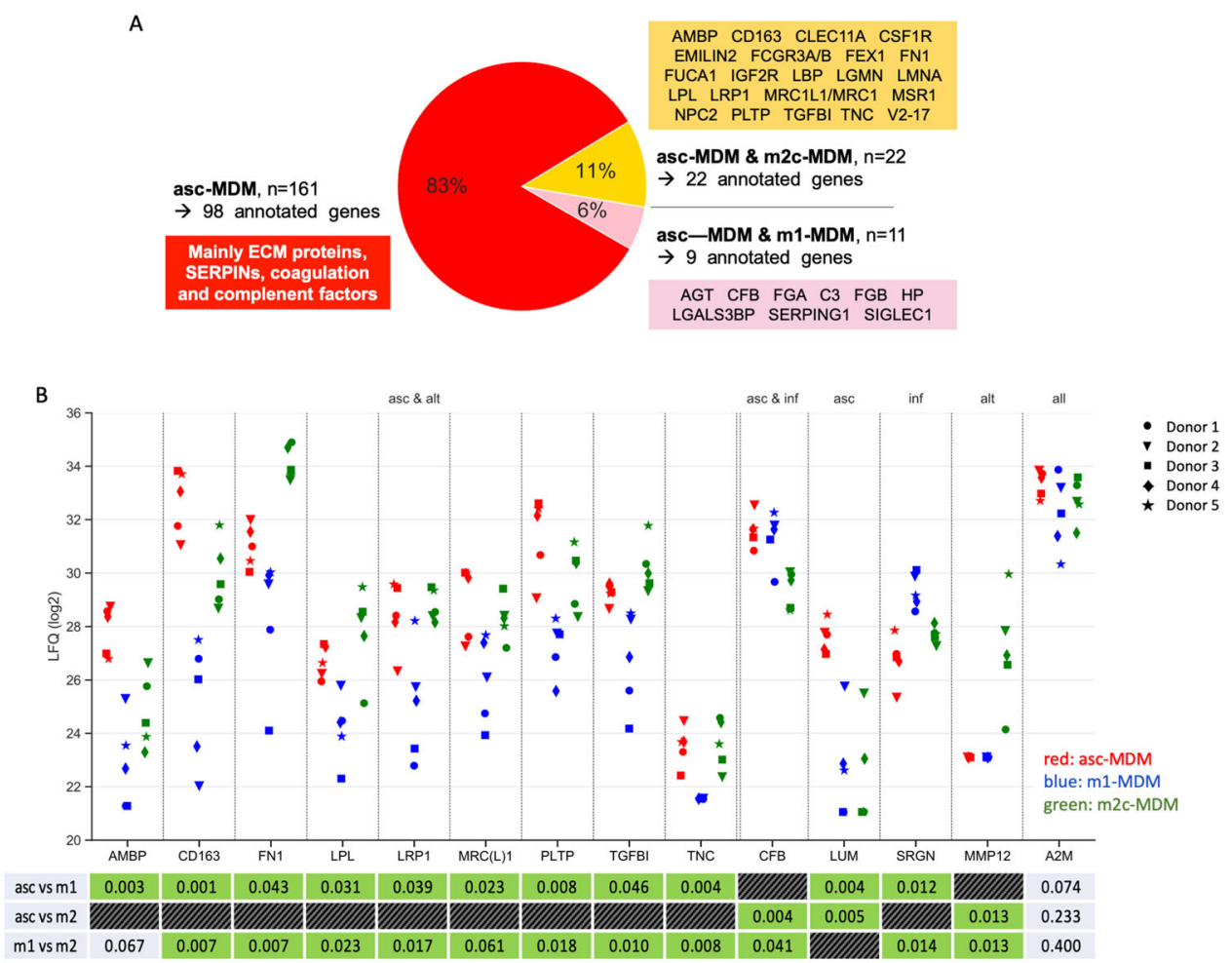

Fig. 2 Secretome analysis of MDM subtypes by LC-MS/MS. Serum-free conditioned media of m1-MDM (induced by LPS+IFNY), m2c-MDM (induced by IL-10), and asc-MDM (induced by ascites) from the same 5 donors tested for stimulation of tumor cell migration in Fig. 1 were analyzed by mass spectrometry-based proteomics. a Pie chart showing the distribution of proteins present selectively in the medium from asc-MDM and $\mathrm{m} 2 \mathrm{c}-$ MDM versus m1-MDM (orange), asc-MDM and m1-MDM versus m2c-MDM (pink), and asc-MDM versus m1-MDM and m2c-MDM (red). Numbers ( $n$ ) refer to the identified polypeptides (feature_ids in Table S1); arrows point to the number of annotated genes that could be associated with the identified polypeptides. The respective genes (or gene functions) are listed in the colored boxes. b Dot plot showing protein levels individually (log 2 LFQ values measured by LC-MS/MS) for MDM from the same donors as in Fig. 1. Arrows indicate the following selectivities: asc \& alt: asc-MDM and m2c-MDM versus m1-MDM (orange in a); asc \& inf: higher level found with asc-MDM and m1-MDM versus m2c-MDM (pink in a); asc: asc-MDM versus m1-MDM and m2c-MDM (red in a); inf: m1-MDM versus asc-MDM and m2c-MDM; alt: m2c-MDM versus m1-MDM and m2c-MDM. The table at the bottom shows $p$ values (paired $t$ test) for the relevant comparisons. Green: $p<0.05$; gray $p \geq 0.05$.

(Table S1). Of these, the 22 proteins were present at higher levels in conditioned media from asc-MDM and $\mathrm{m} 2 \mathrm{c}-\mathrm{MDM}$ compared to $\mathrm{m} 1-\mathrm{MDM}$ in at least four out of five triplets (Fig. 2a; Tables 1 and S2). Nine of these proteins (AMBP, CD163, FN1, LPL, LRP1, MRC1L1/ MRC1, PLTP, TGFBI, TNC) perfectly fit this distribution in all five triplets (Fig. 2b). Among these, three proteins are migration-promoting candidates as suggested by literature data ${ }^{28-30}$, i.e., TGFBI, TNC, and FN1 (Table 1). A similar observation was made for macrophage mannose receptor 1 (MRC1, CD206) and scavenger receptor cysteine-rich type 1 protein (CD163), which are known to be commonly upregulated in TAM and alternatively activated macrophages ${ }^{8,17,25}$, and thus serve as plausibility controls.

We also identified proteins selective for other MDM subtypes, including 9 proteins with annotated genes for asc-MDM and m1-MDM versus m2c-MDM (Table S3; Fig. 2a), as well as 98 proteins for asc-MDM versus both
m1-MDM and m2c-MDM (Table S4; Fig. 2a). This is exemplified in Fig. $2 b$ by lumican (LUM), serglycin (SRGN), and metallopeptidase 12 (MMP12), which are secreted proteins selective for asc-MDM, m1-MDM, or $\mathrm{m} 2 \mathrm{c}-\mathrm{MDM}$. In contrast, alpha-2-macroglobulin (A2M) is a protein present at similar levels in conditioned media from all macrophage subtypes (Fig. 2b).

Intriguingly, the proteins secreted selectively by ascMDM are mainly composed of ECM-associated polypeptides (such as collagens, BCAM, LUM, SERPIN protease inhibitors) as well as complement factors (Table S4; Fig. 2a). This is consistent with previous reports describing these proteins as a hallmark of TAM in HGSC $\operatorname{ascites}^{7,13}$, further validating the experimental approach.

TGFBI, TNC, and FN1 are secreted by ascites TAM in vivo and are associated with a short relapse-free survival (RFS)

To assess the clinical significance of TGFBI, TNC, and FN1, we analyzed their levels in ascites from 70 HGSC 
Table 1 Top 22 secreted proteins overexpressed in the secretomes of asc-MDM and m2c-MDM compared to m1-MDM.

\begin{tabular}{|c|c|c|}
\hline Gene name & Protein name & Match $(n)^{\mathrm{a}}$ \\
\hline AMBP & Alpha-1 microglobulin & 5 \\
\hline CD163 & Scavenger receptor CD163 & 5 \\
\hline FN1 & Fibronectin & 5 \\
\hline LPL & Lipoprotein lipase & 5 \\
\hline LRP1 & LDL receptor-related protein 1 & 5 \\
\hline $\operatorname{MRC}(L) 1$ & Macrophage mannose receptor 1 & 5 \\
\hline PLTP & Phospholipid transfer protein & 5 \\
\hline TGFBI & Transforming growth factor beta induced & 5 \\
\hline TNC & Tenascin & 5 \\
\hline IGF2R & $\begin{array}{l}\text { Cation-independent mannose-6-phosphate } \\
\text { receptor }\end{array}$ & 4 \\
\hline CLEC11A & C-type lectin domain family 11 member A & 4 \\
\hline CSF1R & $\begin{array}{l}\text { Macrophage colony-stimulating factor } 1 \\
\text { receptor }\end{array}$ & 4 \\
\hline EMILIN2 & Elastin Microfibril Interfacer 1 & 4 \\
\hline FCGR3A & Fc fragment of IgG receptor IIIA & 4 \\
\hline FUCA1 & Alpha-1 fucosidase & 4 \\
\hline LBP & Lipopolysaccharide-binding protein & 4 \\
\hline LGMN & Legumain & 4 \\
\hline LMNA & Lamin A/C & 4 \\
\hline MSR1 & Macrophage scavenger receptor 1 & 4 \\
\hline NPC2 & Epididymal secretory protein E1 & 4 \\
\hline STAB1 (FEX1) & Stabilin & 4 \\
\hline V2-17 & Ig lambda chain V-IV region Hil & 4 \\
\hline
\end{tabular}

${ }^{\text {a Match }}(n)=$ number of donors matching the classification of selectivity ( $n$ out of 5).

patients and 30 blood plasma samples in our recently published dataset ${ }^{31}$ obtained by the aptamer-based SOMAscan technology ${ }^{32}$. All three proteins were present at significantly higher levels in ascites compared to plasma from patients of the same cohort $(n=20)$ as well as plasma from patients with benign gynecological diseases $(n=10)(p<0.0001$, Fig. 3a). To elucidate the origin of TGFBI, TNC, and FN1 in HGSC ascites, we made use of our transcriptome, proteome, and secretome datasets for tumor cells, TAMs, and TATs ${ }^{7,33}$. As shown in Fig. 3 for both TGFBI and FN1, RNA expression, intracellular protein levels, as well as secretion were consistently strongest in TAMs compared to tumor cells and TATs (Fig. 3b-d). These findings are consistent with the data reported in the present study showing that asc-MDM secrete TGFBI and FN1. TNC, on the other hand, is also secreted by TAMs at a level comparable to TGFBI (Fig. $3 \mathrm{~d})$, but in the corresponding omics datasets TNC mRNA was very low in TAM (Fig. 3b) and intracellular TNC protein was not detectable (Fig. 3c). This apparent discrepancy was confirmed with in vitro differentiated ascTAM by quantitative reverse transcription polymerase chain reaction (qRT-PCR) and western blotting (see below and Fig. $5 \mathrm{~d}-\mathrm{g}$ ), which may be explained by an unusual instability of TNC mRNA in macrophages combined with rapid protein secretion.

Next, we evaluated the potential clinical significance of TGFBI, TNC, and FN1 by associating their ascites levels (SOMAscan data as above) and RFS in a set of 66 HGSC patients. As illustrated in Fig. 4, Kaplan-Meier plots revealed a significant association with high ascites concentrations of TGFBI (Fig. 4a; logrank $p=0.010$; hazard ratio $(\mathrm{HR})=2.35$ ), TNC (Fig. $4 \mathrm{~b} ; p=0.005 ; \mathrm{HR}=2.99$ ), or FN1 (Fig. 4c; $p=0.016$; HR $=2.10$ ). These findings are consistent with public datasets (Fig. 4d) showing that the expression of TGFBI, TNC, and FN1 mRNA expression in tumor tissue is inversely associated with overall survival (OS) in both database queried, i.e., The Cancer Genome Atlas (TCGA) ${ }^{34}$ and Kaplan-Meier Plotter (KMP) ${ }^{35}$. Both FN1 and TGFBI also showed an association with a short OS in the PRECOG database ${ }^{36}$.

\section{Validation of TGFBI and TNC secretion by asc-MDM and m2c-MDM}

In agreement with our data, TAM isolated from human tumors have been reported to express a matrix-related signature including FN1 affecting tumor cell motility ${ }^{27}$, whereas a role of TGFBI and TNC in the crosstalk between macrophages and tumor cells has not been addressed in previous studies. We therefore focused our work on these two mediators. To validate and extend the proteomics data in Fig. 2b, which showed increased TGFBI and TNC secretion by asc-MDM and m2c-MDM versus m1-MDM, we applied qRT-PCR, western blot, and enzyme-linked immunosorbent assay (ELISA).

As illustrated in Fig. 5, TGFBI mRNA (Fig. 5a) was significantly higher in $\mathrm{m} 2 \mathrm{c}-\mathrm{MDM}$ compared to both ascMDM and m1-MDM but was similar among the latter two MDM subtypes. At the intracellular protein level, TGFBI was weak or even undetectable in $\mathrm{m1-MDM}$ and $\mathrm{m} 2 \mathrm{c}-\mathrm{MDM}$ but higher in asc-MDM in single donors (Fig. $5 b)$. TGFBI secretion was strongest in m2c-MDM but was also elevated in asc-MDM versus m1-MDM (Fig. 5c), which is consistent with the MS data (Fig. 2b). It thus appears that the differences in TGFBI secretion observed among the three MDM subtypes do not solely result from differential regulation of mRNA expression but also from subtype-specific effects on secretion itself.

TNC mRNA expression (Fig. 5d) were similar in ascMDM and m1-MDM but elevated in m2c-MDM (though 

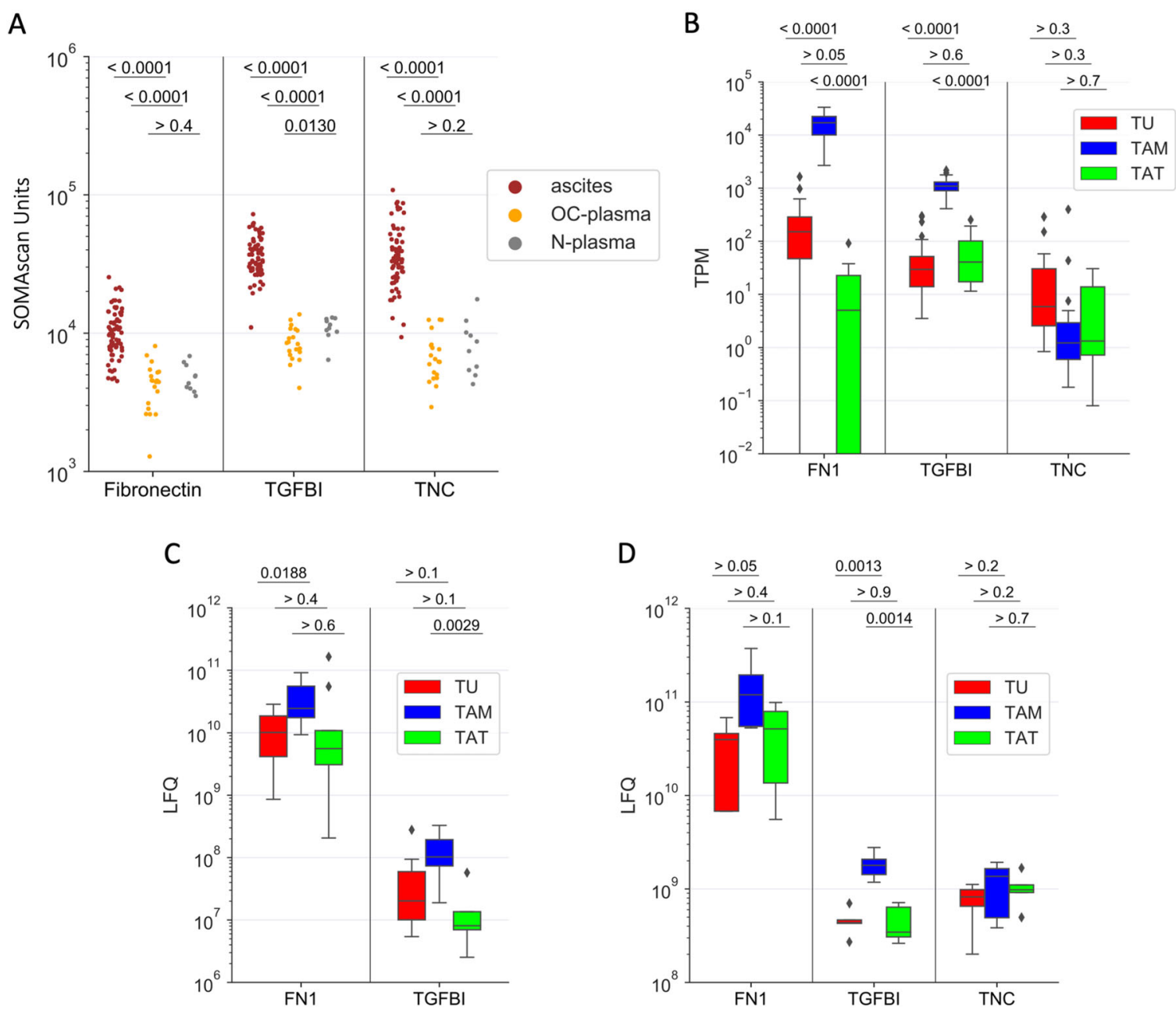

Fig. 3 Expression of TGFBI, TNC, and FN1 in malignant ascites and ascites-associated cells. a Levels (LC-MS/MS, LFQ intensity) of TGFBI, TNC, and FN1 in cell-free HGSC ascites ( $n=70$, red dots), plasma from HGSC patients ( $n=20$; OC-plasma, yellow), and patients with benign gynecologic diseases ( $n=10$; N-plasma, gray) as determined by SOMAscan ${ }^{31}$. b Expression levels (RNA-Seq, TPM values) for TGFBI, TNC, and FN1 in ascitesassociated tumor cells (TU $n=23$, depicted in red), TAM $(n=32$; blue), and TATs ( $n=8$; green). $\mathbf{c}$ Intracellular protein levels (LFQ intensity) of TGFBI, TNC, and FN1 in tumor cells (TU), TAM, and TATs from HGSC patients as obtained from LC-MS/MS-based proteome analysis $\left(n=5\right.$ for each cell type) ${ }^{7}$. d Levels of TGFBI, TNC, and FN1 (LFQ intensity) in the conditioned media of primary tumor cells (TU), TAM, and TATs after a 5-h cultivation in proteinfree medium ( $n=5$ for each cell type). Boxplots show medians (horizontal line in boxes), upper and lower quartiles (box), and range (whiskers) (b-d). Statistical analyses were performed by unpaired $t$ test; $p$ values are shown at the top of each panel.

$p>0.05$ due to the very low expression), whereas intracellular TNC was only detectable in asc-MDM (Fig. 5e). As for TGFBI, TNC secretion was also higher in asc-MDM and m2c-MDM versus m1-MDM (Fig. 5f, g), pointing to a similar subtype-dependent regulation of the secretory pathway.

In summary, these analyses fully confirm the MS data and suggest that differential regulation of both gene expression and secretion are responsible for the differences in TGFBI and TNC secretion by MDM subtypes.

\section{Pro-migratory effects are impaired by neutralizing TGFBI and TNC in the MDM secretome}

We next addressed the impact of TGFBI and TNC secreted by macrophages on tumor migration. TGFBI and
TNC both bind to integrins and, in case of TNC, additionally to EGF receptors present on the surface of tumor cells, thereby activating migration-inducing pathways ${ }^{28,29,37-39}$. We could demonstrate that patient-derived tumor cells selectively bind to rTGFBI or rTNC (full-length protein) but not to rTNC-EGFL, which is a smaller fragment harboring EGFL repeats but lacking integrin-binding domains (Fig. 6a). As illustrated in Fig. 6b, rTGFBI as well as both rTNC forms enhanced migration of OCMI cells, which was accomplished by pre-incubating OCMI cells with the recombinant proteins prior to setting up the transwell assay with fetal calf serum (FCS) as chemoattractant. Our findings thus indicate that, in the case of TNC, integrin interaction is required for adhesion but dispensable for migration. 

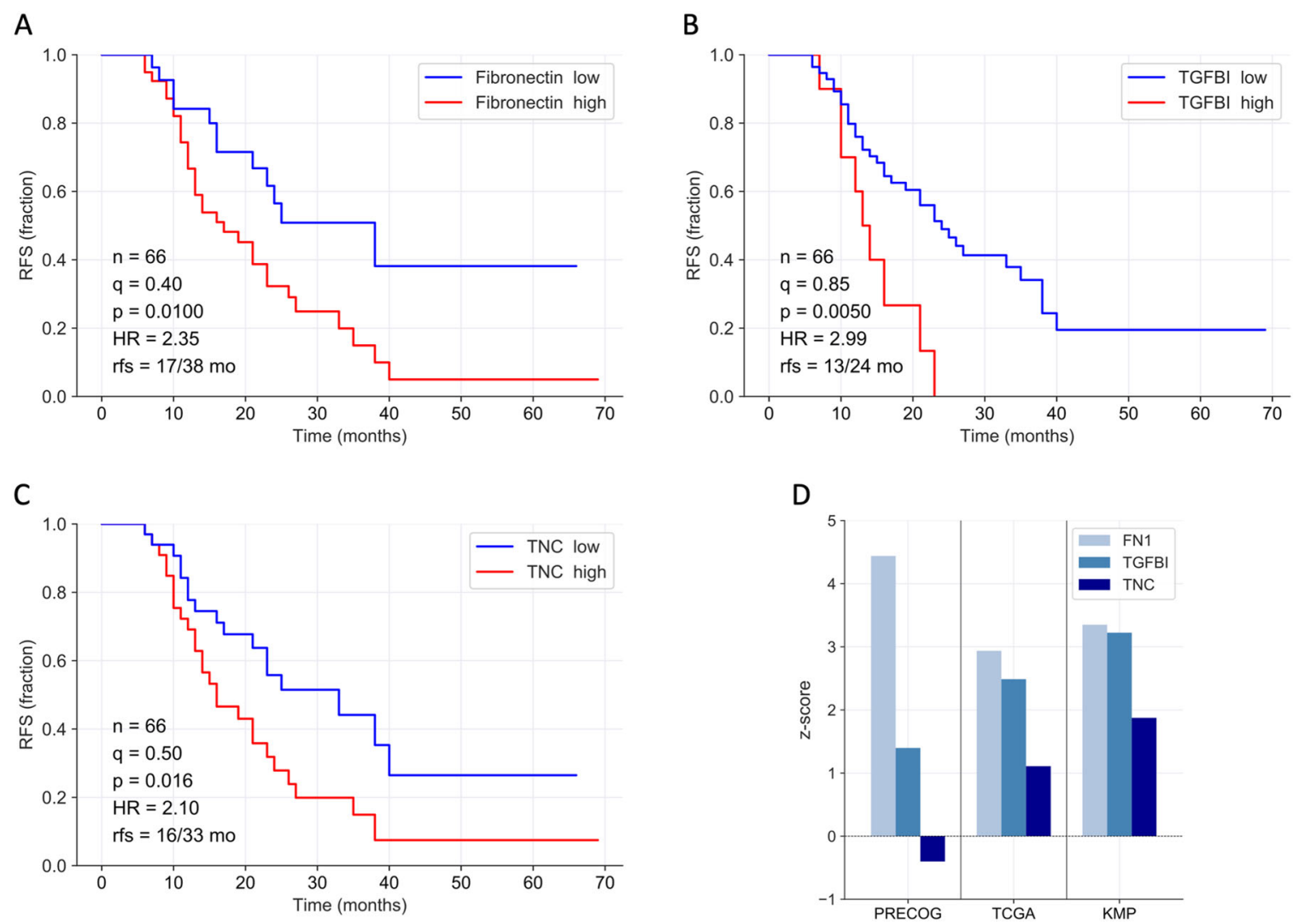

Fig. 4 Association of TGFBI, TNC, and FN1 ascites levels with ovarian cancer survival. a-c Kaplan-Meier plots showing the relationship between relapse-free survival (RFS) and SOMAscan protein signals for fibronectin (a), TGFBI (b), and TNC (c) in cell-free ascites from HGSC patients. $n$ : number of evaluable patients; $q$ quantile used for splitting datasets (high versus low levels), $p$ logrank $p$ value, HR hazard ratio, rfs median RFS (months). d Mean z-scores for survival associations with TGFBI, TNC, and FN1 gene expression in solid tissue from ovarian carcinoma) based on public datasets. TCGA ${ }^{34}$ and KMP ${ }^{35}$ : relapse-free survival (RFS); PRECOG $^{36}$ : overall survival (OS). Positive and negative $z$-scores indicate $H R>1$ and $H R<1$, respectively. A $z$-score of 1.96 corresponds to a logrank $p$ value of 0.05 .

To assess the relevance of TGFBI and TNC in the context of the TAM secretome, we analyzed the effect of neutralizing antibodies directed against these proteins. As a proof of principle, we found that tumor migration induced by rTGFBI and rTNC was blocked by neutralizing anti-TGFBI (Supplementary Fig. S3A, B) and anti-TNC antibodies (Supplementary Fig. S3C, D). More importantly, similar results were obtained when conditioned media from asc-MDM or m2cDM were pre-incubated with the neutralizing antibodies against TGFBI (Fig. 6c, d) and TNC (Fig. 6e, f). In both cases, a significant reduction of cellular migration compared to untreated or IgG control-treated conditioned media was found for five different donors. In conclusion, these data indicate that TNC and TGFBI as constituents of the TAM secretome promote tumor cell migration.

\section{Small interfering RNA (siRNA)-mediated TGFBI silencing in m2c-MDM/asc-MDM blocks tumor migration}

To validate our findings by an independent experimental approach, we made use of siRNA-mediated interference. We focused on TGFBI because of the low expression of TNC mRNA and TNC protein (Fig. 5d, e), which makes it difficult to reliably monitor silencing efficacy. TGFBI silencing was performed in asc-MDM and m2c-MDM and achieved reduction of TGFBI RNA and intracellular TGFBI protein expression by TGFBI siRNA transfection relative to control siRNA (Supplementary Fig. S4A, B). Importantly, TGFBI secretion by asc-MDM and $\mathrm{m} 2 \mathrm{c}-\mathrm{MDM}$ was also inhibited by TGFBI siRNA compared to untransfected $(p<0.05)$ and control siRNA-transfected MDM $(p<0.01)$ (Fig. 7a). To investigate the functional impact of TGFBI knockdown on the migration-inducing capacity of asc-MDM and m2c$\mathrm{MDM}$, transwell assays were performed analogous to the neutralizing experiments above. As shown in Fig. 7b, c, the conditioned media from untransfected or control siRNAtransfected asc-MDM and m2c-MDM induced OCMI cell migration to a very similar extent, whereas transfection with TGFBI siRNA resulted in a reduced effect. Taken together, these results establish an essential role for TGFBI as a migration-promoting factor in the TAM secretome. 


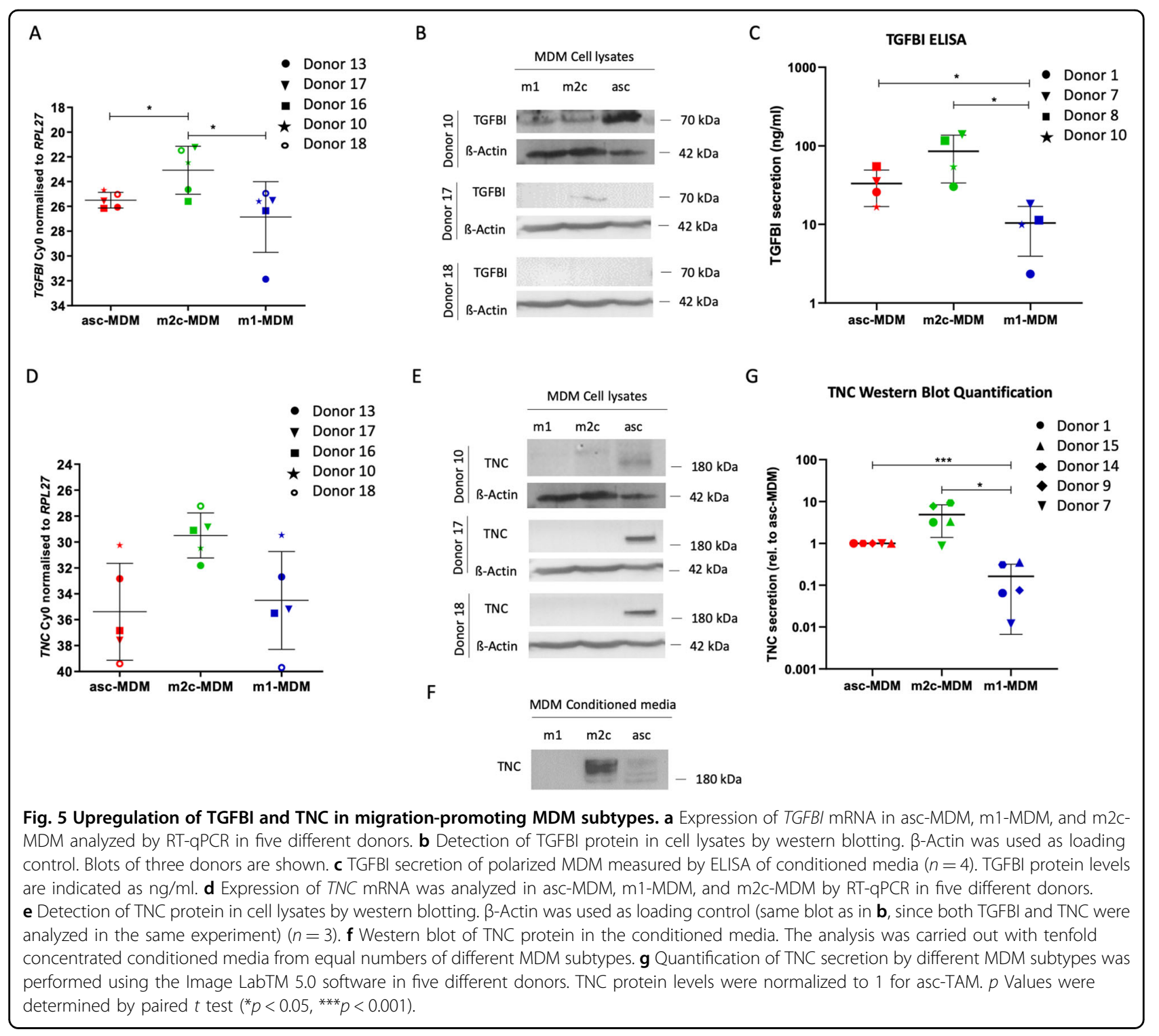

\section{Discussion}

In the present study we identified TGFBI, TNC, and FN1 as potential mediators of TAM-induced ovarian cancer migration, underscoring the known role of ECM proteins in tumor progression. A clinical importance of all three proteins is supported by our finding that increased ascites level is associated with a short RFS (Fig. $4 a-c$ ). Consistent with this observation, we found a similar association of FN1, TGFBI, and TNC gene expression in solid tumor tissue with a poor clinical outcome (Fig. 4d). These findings are in line with studies on colorectal cancer and esophageal squamous cell carcinoma where a poor prognosis correlates with TGFBI expression in tumor stroma ${ }^{40,41}$. For TNC, similar clinical associations have been reported for different tumor entities ${ }^{42-45}$. Furthermore, FN1, TNC, and TGFBI have been reported to promote tumor migration, invasion, and adhesion, which are functions facilitating metastatic spread ${ }^{28-30}$. In this context, FN1 has been proposed as a promoter of ovarian cancer released by $\mathrm{CAFs}^{45}$ and $\mathrm{TAMs}^{27}$, a hypothesis our results confirm. On the other hand, a mechanistic link of TAM-secreted TNC and TGFBI with tumor migration, as identified in our study, has not been described as of yet.

TGFBI often functions as a linker protein to interconnect ECM molecules and induces cell interactions through integrins ${ }^{37,46-49}$. Different physiological functions including migration and adhesion have been attributed to TGFBI $^{37,50-52}$. Previous work has shown that TGFBI is expressed by stromal fibroblasts and cancer cells ${ }^{53}$. TGFBI upregulation in M2 macrophages has also been linked to acute inflammation processes and ECM remodeling ${ }^{51}$, but 


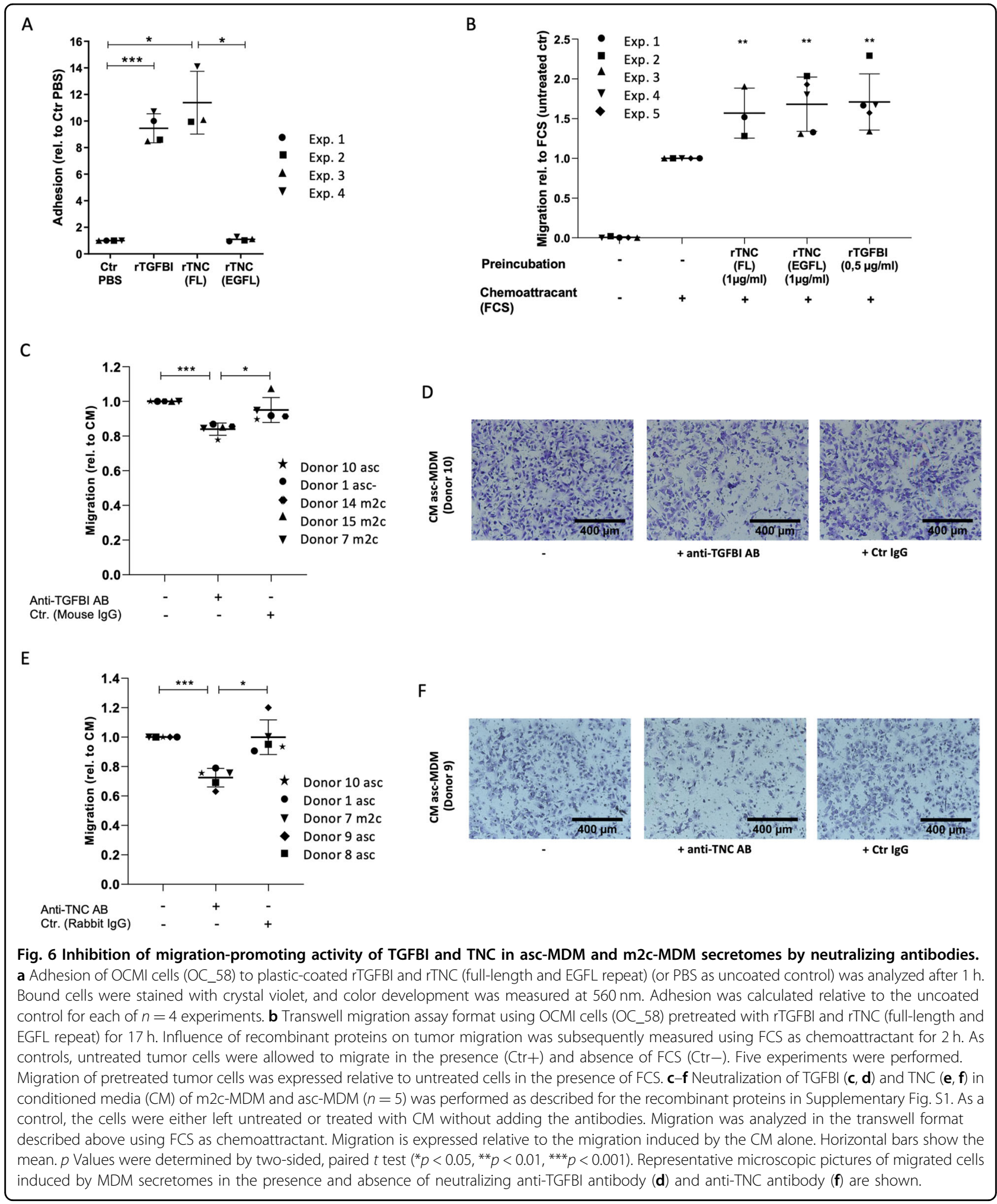

TAMs have not been identified as producers of TGFBI to date.

In ovarian cancer and esophageal squamous cell carcinoma, a dual function of TGFBI depending on its cellular origin has been discussed ${ }^{38,41,54}$. Here TGFBI has been proposed to act as tumor suppressor as it is downregulated in tumor cells and as tumor promoter when expressed by peritoneal stroma cells. In accordance with 

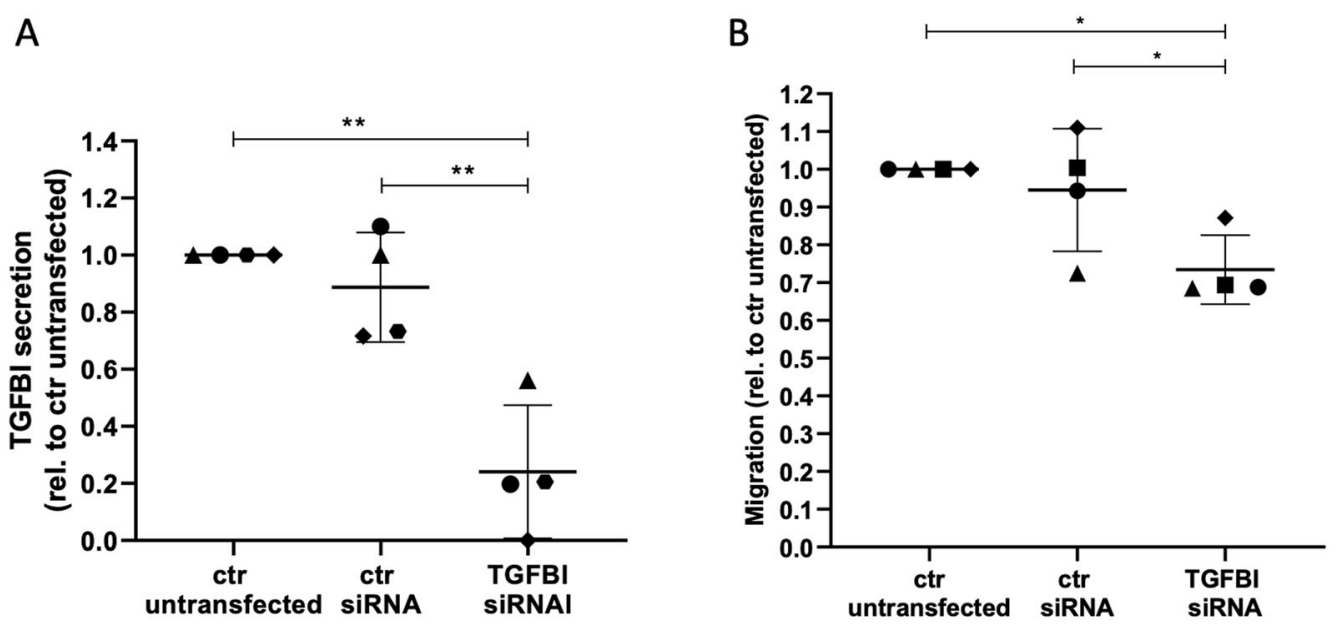

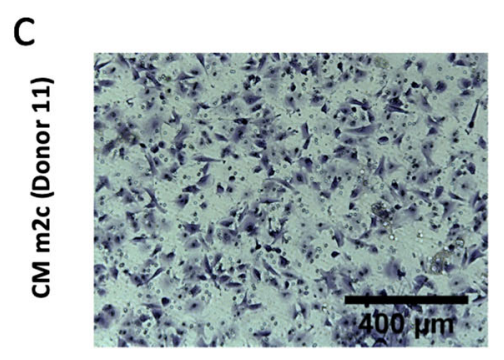

Ctr untransfected

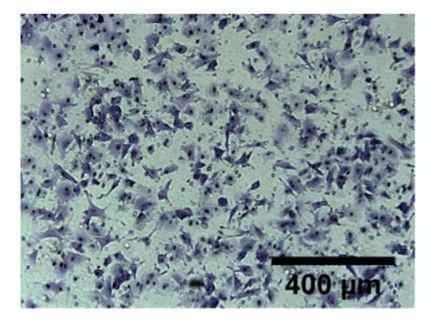

Ctr siRNA

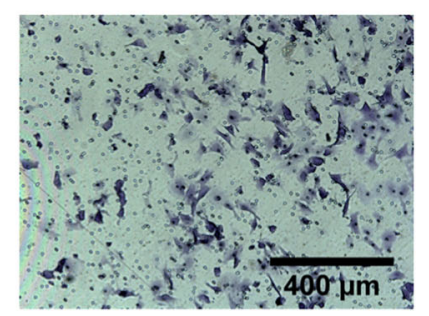

TGFBI SiRNA

Fig. 7 Impact of TGFBI silencing on the migration-promoting potential of asc-MDM and m2c-MDM secretomes. a TGFBI secretion by $\mathrm{m} 2 \mathrm{C}$ MDM and asc-MDM after siRNA-mediated TGFBI silencing. TGFBI concentration in the conditioned media of MDM transfected with control siRNA and TGFBI siRNA (pool of three siRNAs) was determined by ELISA and normalized to the untransfected control. Depicted are the data of five different macrophage preparations. Additional data of TGFBI gene expression and intracellular protein levels in TGFBI siRNA-transfected macrophages are shown in Fig. S3. $\mathbf{b}$ Influence of TGFBI knockdown on the migration-promoting potential of asc-MDM and m2C-MDM. OCMI tumor cells (OC_58) were pretreated with conditioned media of the untransfected and siRNA-transfected cells before applied to a transwell migration assay with FCS as attractant, as described in the legend of Fig. 6. Migration was expressed relative to the untransfected control for each of the four different macrophage preparations. Horizontal bars show the mean and two-sided, paired $t$ test was calculated $\left({ }^{*} p<0.05,{ }^{* *} p<0.01\right)$.

c Representative microscopic pictures of tumor cell migration induced by conditioned media from m2c-MDM (donor 11) untransfected or transfected with control siRNA or TGFBI siRNA.

these reports, we demonstrated that TAM secrete higher amounts of TGFBI compared to other cell populations in the ascites, e.g., tumor cells or TATs (Fig. 3), and that TGFBI in the TAM secretome enhances tumor migration proven by neutralizing antibodies (Fig. 6) and RNA silencing (Fig. 7).

Both anti- and pro-adhesive features have been attributed to TGFBI affecting cell motility and invasion. In the case of melanoma, TGFBI exhibits anti-adhesive properties concomitant with anti-migratory activity ${ }^{55,56}$, whereas TGFBI mediates adhesion and migration in renal cell carcinoma ${ }^{57}$. Judging from our data, TGFBI seems to have a pro-adhesive effect in HGSC, since primary OCMI cells adhere strongly to rTGFBI, which is accompanied by enhanced tumor motility (Fig. 6).

Similar to TGFBI, TNC also functions as a modulator of cell adhesion and migration, but a broad range of functions linked to different TNC isoforms $(180-330 \mathrm{kDa})$ have been reported beyond these ${ }^{58,59}$. TNC is downregulated in healthy tissue but transiently reexpressed under pathological conditions like inflammation, wound healing, and cancer ${ }^{58,60}$. Moreover, TNC was found at the invasive front of different tumors with CAFs being the main producers ${ }^{61-63}$. For macrophages, TNC secretion has so far only been shown in atherosclerotic plaques $^{64}$. In the present study, we identify TAM as a cellular origin of TNC in the ovarian TME supporting tumor migration. As shown by western blot, migrationpromoting macrophages predominantly secrete large TNC variants of about $200-250 \mathrm{kDa}$ (Fig. 5f), which have been proposed to promote a tumor-supporting $\mathrm{TME}^{59}$. Interestingly, a number of studies point to an association between cancer progression and the occurrence of large TNC isoforms harboring alternatively spliced FNIII repeats ${ }^{59,65}$. Alternatively spliced FNIII repeats as well as the RGD-containing FNIII 3 repeat present in TNC isoforms mediate cell adhesion via interaction with different 
integrins expressed on the surface of tumor cells ${ }^{58,66-68}$. Our data showing that only a full-length rTNC promoted cellular attachment, whereas a smaller fragment lacking integrin-binding sites exhibited anti-adhesive properties (Fig. 6a) may be considered to generally support that assumption. By contrast, both rTNC equally induced tumor migration indicating that the EGFL repeats present in both TNC forms might be involved in tumor migration through activation of EGF receptor signaling.

TGFBI and TNC are both induced by TGF $\beta$ signaling and share common binding partners like fibronectin, collagen, and proteoglycans ${ }^{37,69}$. Moreover, both proteins bind to integrins expressed on tumor cells and mediate their function via the integrin signaling pathway ${ }^{67}$. These similarities may contribute to a functional cooperation of TGFBI and TNC in mediating tumor migration, when secreted by TAMs and other cells in the TME.

Other factors, including EGF, IGF1, and CHI3L1, have been proposed to be involved in the migration-promoting function of macrophages ${ }^{22-24}$, but these mediators are not among the proteins upregulated in the pro-migratory MDM secretomes identified by our approach. Several reasons are likely to contribute to these differences.

(i) Our strategy was to identify proteins that are selectively secreted by migration-promoting ascMDM and m2c-MDM relative to m1-MDM, which do not impact tumor migration. CHI3L1 is actually present in the secretomes from all three MDM subtypes, albeit with elevated levels for m1MDM (Table S2), which, however, does not preclude a contribution of CHI3L1 to tumor cell migration as seen in other studies ${ }^{24}$.

(ii) The published studies differ from ours in that they use either the THP1 macrophage cell line or MDM differentiated to M2a cells by IL-4, which are likely to secrete different factors.

(iii) We consider only proteins to be relevant that are produced by TAM in vivo. EGF and IGF1 are both not expressed by TAM from HGSC ascites (median transcripts per kilobase million $(\mathrm{TPM})<0.1)$ or at very low levels (median $\mathrm{TPM}=1$ ), respectively (https://www.ovara.net/resources) ${ }^{7}$. Both proteins were neither detectable in the intracellular proteome of TAM nor in their secretome ${ }^{7}$ and were not found in the conditioned medium from ascTAM (and this study, Table S2). It therefore appears unlikely that EGF and IGF1 play a role in the HGSC TME as proteins secreted by TAMs. However, both proteins are present in the ascites proteome of HGSC patients $^{31}$, pointing to other cell types as producers of EGF and IGF1. Therefore, a role of these proteins in tumor migration and HGSC metastasis cannot be ruled out.
In conclusion, TGFBI, TNC, and FN1, predicted by our experimental model as migration-promoting proteins secreted by TAMs were validated to be (i) present in the HGSC ascites, (ii) secreted by TAMs derived from ascites, (iii) associated with a poor clinical outcome, and (iv) promote tumor migration as part of the TAM secretome. These findings provide further evidence for the essential role of TAMs and the ECM in HGSC metastasis.

\section{Materials/subjects and methods}

\section{Ascites and cells isolated from ovarian cancer patients}

Ascites was collected from untreated patients with HGSC prior to surgery at Marburg University Hospital. The collection and analysis of human material were approved by the ethics committee at Philipps University (reference number 205/10). Donors provided written consent in accordance with the Declaration of Helsinki. TAMs, TATs, and tumor cells were isolated from ascites as previously described ${ }^{7,33}$. Briefly, mononuclear cells were separated by Lymphocyte Separation Medium 1077 (PromoCell, Heidelberg, Germany) density gradient centrifugation followed by MACS separation of CD14+ TAMs and CD3+ TATs and purification of tumor spheroids by size exclusion. Cell-free ascites was cryopreserved at $-80^{\circ} \mathrm{C}$. Permanent primary tumor cell cultures (termed OCMI tumor cells) were established from ascites tumor spheroids according to Ince et al. ${ }^{70}$ with modifications, as previously reported ${ }^{71}$. This culture system allows for the propagation of ovarian cancer cells over long periods of time in the absence of cultureinduced crisis or genetic alterations as compared to the original tumor. Cultured HGSC patient-derived OCMI cell lines (OCMI OC_37, OC_38, OC_58, OC_92, and OC_108) were tested for mycoplasma contamination before use for functional analysis.

\section{Isolation and culture of MDMs from healthy donors}

Buffy coats from healthy adult volunteers were kindly provided by the Center for Transfusion Medicine and Hemotherapy at the University Hospital Gießen and Marburg, and mononuclear cells were isolated by Ficoll density gradient centrifugation. CD14+ monocytes were purified by adherence selection and used for subsequent differentiation at a concentration of approximately $2.5 \times 10^{6}$ cells per 6-well plate. For differentiation into TAMs like asc-MDM, monocytes were cultured in cell-free ascites pool derived from 5 patients for 7 days. m1-MDM and m2c-MDM were generated by culturing monocytes in RPMI1640 (Life Technologies, Darmstadt, Germany) supplemented with 5\% human AB serum (Sigma), 1\% sodium pyruvate (Sigma Aldrich, Taufkirchen, Germany), and $100 \mathrm{ng} / \mathrm{ml}$ granulocyte macrophage colony-stimulating factor (CSF) (Peprotech, Hamburg, Germany) for m1-MDM or $20 \mathrm{ng} / \mathrm{ml}$ macrophage CSF (MCSF; Biolegend, San Diego, CA, USA) for m2c-MDM ${ }^{25}$. 
After 5 days, $100 \mathrm{ng} / \mathrm{ml}$ LPS (Sigma Aldrich, Taufkirchen, Germany) and $20 \mathrm{ng} / \mathrm{ml}$ IFN $\gamma$ (Biozol, Echingen, Germany) was added for m1-MDM and $20 \mathrm{ng} / \mathrm{ml}$ IL-10 (Biozol, Echingen, Germany) for m2c-MDM activation for 2 days.

\section{Flow cytometry}

The differentiation phenotype of MDM was characterized by flow cytometry (FACSCanto II BD Biosciences) as described previously ${ }^{8}$ using the following antibodies for surface staining: anti-human CD14-FITC (5170518160, Miltenyi Biotec, Bergisch Gladbach, Germany), CD86-FITC (5170620163, Miltenyi Biotec), CD16-PE-Cy7 (4273442, eBioscience, Frankfurt, Germany), CD163-PE (4303842, eBioscience), HLA-DR-APC (4330406, eBioscience), CCR7PE (5247917, BD Biosciences, Heidelberg, Germany), and CD206-APC (B202691, Biolegend). Corresponding isotypematched controls were purchased from Miltenyi Biotec (5161221581; 5161017246) and BD Biosciences (6286946; 25471442). The gating for macrophages was performed based on the surface expression of CD14 marker. Results were calculated as the percentage of positive cells and mean fluorescence intensities.

\section{Generation of conditioned media}

For the proteomic analysis in Fig. 3, conditioned media from ascites-derived tumor spheroids, TAMs, and TATs were generated as described by Worzfeld et al. ${ }^{7}$. Conditioned media of TAMs were also used for tumor migration assays (Fig. 1). Therefore, freshly prepared TAMs were cultured in autologous cell-free ascites (or ascites pool of five patients) at a density of $2.5 \times 10^{6}$ cells per 6 -well plate for $16 \mathrm{~h}$ at $37^{\circ} \mathrm{C}$ and $5 \% \mathrm{CO}_{2}$. Thereafter, the ascites was aspirated, and the cells were washed three times in phosphate-buffered saline (PBS) and twice in serum-free media M199 (Gibco, Thermo Fisher Scientific, Schwerte, Germany) mixed 1:2 with Dulbecco's Modified Eagle's Medium/Ham's F-12 (1:1 Biochrom, Berlin, Germany). TAMs were cultured in medium (750 $\mu \mathrm{l}$ per 6-well) without ascites or serum for another $5 \mathrm{~h}$ at $37^{\circ} \mathrm{C}$ and $5 \% \mathrm{CO}_{2}$ before collecting the conditioned media for secretome analysis and functional testing. This time point was chosen as prolonged incubation of TAMs resulted in increased cell death as shown by lactate dehydrogenase release. Conditioned media from differentiated MDM were obtained analogously, except that MDM were cultured for $18 \mathrm{~h}$ in serum-free medium. For immunoblotting, conditioned media were concentrated tenfold using a vacuum concentrator.

\section{Proteomic and transcriptomic analyses}

Cell culture supernatants from MDM cultures was obtained as described above. Up to $40 \mu \mathrm{g}$ of proteins were loaded on a gradient gel (NuPAGE 4-12\% Bis-Tris gel, Invitrogen, Carlsbad, CA, USA), separated by sodium dodecyl sulfate-polyacrylamide gel electrophoresis prior to in-gel digestion ${ }^{72}$ and analyzed by liquid chromatography tandem MS/MS as previously reported ${ }^{7}$. The proteomics data have been deposited to the ProteomeXchange Consortium via the PRIDE partner repository $^{73}$ at www.ebi.ac.uk/pride/archive (dataset identifier PXD016555). Data were processed as described ${ }^{7}$ using the human uniProt database (canonical and isoforms, downloaded on 02/09/2018, 183579 entries). Relevant parameters for instrumentation extracted using MARMoSET $^{74}$ and are, along with MaxQuant ${ }^{75-77}$ (v. 1.6.1.0) parameters, included in Supplementary Materials. Transcriptomic and proteomic data for TAMs, TATs and tumor cells from ascites were derived from our published datasets $^{7,33}$.

\section{Identification of secreted proteins selective for MDM subtypes}

MS data were filtered to include only proteins detected in at least 1 of the 5 asc-MDM samples with a minimum $\log _{2}$ LFQ of 22 (corresponding to the median of the entire dataset with missing values replaced by imputation). Differences between asc-MDM, m1-MDM, and m2cMDM were determined for each protein and triplet. Proteins were considered subtype-selective if they were present in the medium from one MDM subtype at a higher level than in the culture supernatant from another subtype in at least four out of the five triplets.

\section{Tumor cell migration}

Transwell migration assays were performed using two different formats. In a first approach, the migration of primary OCMI tumor cells was determined in the presence of conditioned media of macrophages or recombinant human rTGFBI (R\&D Systems, Wiesbaden, Germany) and rTNC (fragment containing the EGFL repeats: R\&D Systems; fulllength protein: Merck, Darmstadt, Germany) as chemoattractant. 50,000 tumor cells were seeded in $300 \mu \mathrm{l}$ serum-free OCMI medium per transwell insert $(8.0 \mu \mathrm{m}$ pore size; BD Biosciences). Conditioned media of macrophages (1:3 diluted in serum-free OCMI) and rTGFBI $(0.5-5 \mu \mathrm{g} / \mathrm{ml})$ and rTNC $(1-10 \mu \mathrm{g} / \mathrm{ml}$ ) (or $5 \% \mathrm{FBS}$ as positive control) in serum-free OCMI medium were added as chemoattractants to the lower chamber. The cells were allowed to migrate through the filter for $17 \mathrm{~h}$ at $37^{\circ} \mathrm{C}$ in a $5 \% \mathrm{CO}_{2}$ incubator. Filters were stained with crystal violet solution $(0.2 \%$ in $20 \%$ methanol, $1: 5$ dilution) for $10 \mathrm{~min}$ and evaluated under a Leica DMI3000B microscope (Leica, Wetzlar, Germany). Migrated cells were counted in seven visual fields per filter using the ImageJ software. In a second setting, OCMI tumor cells were preincubated with conditioned media of macrophages (1:3 diluted in OCMI medium) and recombinant proteins for $17 \mathrm{~h}$ at $37^{\circ} \mathrm{C}$ and $5 \% \mathrm{CO}_{2}$ prior to performing transwell migration assays with 5\% FCS as chemoattractant. Where indicated, neutralizing antibodies $(10 \mu \mathrm{g} / \mathrm{ml})$ directed against 
TGFBI (3054632, Proteintech, Manchester, UK) and TNC (10000035, Merck)—or equivalent amounts of speciesmatched rabbit (I5006-10MG, Sigma Aldrich) and mouse IgG (131515, Jackson Immuno Research, Cambridgeshire, UK) as controls-were added to conditioned macrophage medium or recombinant proteins for $1 \mathrm{~h}$ before applying to the tumor cells. In each case, the pretreated tumor cells were allowed to migrate for $2 \mathrm{~h}$ and analyzed as described above.

\section{Tumor cell adhesion to TNC and TGFBI}

Ninety-six-well plates were coated in triplicates with $10 \mu \mathrm{g} /$ $\mathrm{ml}$ rTGFBI and rTNC in PBS (or PBS alone as negative control) overnight at $4{ }^{\circ} \mathrm{C}$. Wells were blocked with $1 \%$ bovine serum albumin in PBS for $1 \mathrm{~h}$ at $37^{\circ} \mathrm{C}$ and washed three times with PBS. Fifty thousand tumor cells in OCMI media were added per well and allowed to adhere for $2 \mathrm{~h}$ at $37^{\circ} \mathrm{C}$. The wells were washed with PBS twice to remove any unbound cells. Adherent cells were fixed with glutaraldehyde and stained with $0.1 \%$ crystal violet as described ${ }^{78}$. The photometric measurement was performed at $560 \mathrm{~nm}$, and cell adhesion was expressed relative to the negative control.

\section{TGFBI quantification by ELISA}

TGFBI concentrations in conditioned media of macrophages were quantified by commercial ELISA (human $\beta I G-H 3$ ELISA duo set, R\&D Systems, Wiesbaden, Germany) according to the instructions of the manufacturer.

\section{Immunoblotting and protein quantification}

Immunoblots were performed according to standard western blotting protocols using the following antibodies: $\alpha$-TGFBI (5601, Cell Signaling, Danvers, MA, USA), $\alpha-$ TNC (10000035, Merck), $\alpha$ - $\beta$-Actin (A5441, Sigma Aldrich), $\alpha$-rabbit IgG horseradish peroxidase (HRP)linked AB (27, Cell Signaling), and $\alpha$-mouse IgG HRPlinked AB (32, Cell Signaling). Imaging and quantification was done using the ChemiDoc MP system and Image Lab software version 5 (Bio-Rad, Hercules, CA, USA).

\section{RNA isolation and RT-qPCR}

CDNA isolation and qPCR analyses were performed as described previously ${ }^{79}$. L27 was used for normalization. RT-qPCR was carried out using the following primers: RPL27, AAAGCTGTCATCGTGAAGAAC and GCTGT CACTTTGCGGGGGTAG; TGFBI, AAAGACATCCTA GCCACCAACG and AGCTGGCCTCTAAGTATCTG TACC; and TNC, GCCTCCACAGCCAAAGAACC and TCTGGTGCTGAACGAACTGC. Raw data were evaluated with the $\mathrm{Cy} 0$ method $^{80}$.

\section{siRNA transfection of macrophages for transient TGFBI knockdown}

siRNA transfection was performed in m2c-MDM and TAM-like differentiated asc-MDM as well as ascites- derived TAMs according to the manufacturer's protocol using the TransIT-X2 reagent from Mirus (Madison, WI, USA). The following equimolar mixtures of three siRNA oligonucleotides each from Sigma Aldrich (Taufkirchen, Germany) were used for transfection: siRNA TGFBI \#1 (HA12627314; HA12627315), siRNA TGFBI \#2 (HA12627318; HA12627319), and siRNA TGFBI \#3 (HA12627316; HA12627317). MISSION siRNA Universal Negative Control \# 2 from Sigma Aldrich was used as a control siRNA (si-ctrl). For m2c-MDM, transfection was performed in $\mathrm{RPMI} / 5 \% \mathrm{AB}$-media containing $\mathrm{M}-\mathrm{CSF}$ and IL-10. Since ascites interferes with siRNA transfection, ascites-containing culture medium in asc-MDM or TAM was replaced by $\mathrm{RPMI} / 5 \% \mathrm{AB}$-media during transfection. In this case, after $6 \mathrm{~h}$ transfection medium was changed and ascites was added again to maintain the TAM-like phenotype. Cells were harvested $48 \mathrm{~h}$ after transfection for analysis of RNA/protein expression and generation of conditioned media for functional assays.

\section{Statistical analysis}

Comparative data were statistically analyzed by unpaired (Fig. 3) or paired Student's $t$ test (Figs. 1, 2, 5-7) (two-sided, equal variance). Significance levels are indicated as four $(* * * *)$, three $(* * *)$, double $(* *)$ and single $(*)$ asterisks for $p<0.0001, p<0.001, p<0.01$, and $p<0.05$, respectively. Box pots in Fig. 3 depicting medians (line), upper and lower quartiles (box), range (whiskers), and outliers/fliers (diamonds) were constructed using the Seaborn boxplot function with Python. Associations with RFS (logrank test), HR, and median survival times were analyzed using the Python Lifelines KaplanMeierFitter and CoxPHFitter functions. All logrank test results are presented as nominal $p$ values. The data in Fig. $4 \mathrm{~d}$ were obtained from the PRECOG and KMP meta-analysis databases $^{35,36}$ and TCGA ${ }^{34}$.

\section{Acknowledgements}

We are grateful to Traute Plaum-Allmeroth, Achim Allmeroth, and Sylvia Jeratsch for expert technical assistance. This work was supported by a grant from the German Cancer Aid (Deutsche Krebshilfe) to R.M. and S.R.

\footnotetext{
Author details

${ }^{1}$ Institute of Molecular Biology and Tumor Research (IMT), Center for Tumor Biology and Immunology, Philipps University, Marburg, Germany. ${ }^{2}$ Clinic for Gynecology, Gynecologic Oncology and Endocrinology, Center for Tumor Biology and Immunology, Philipps University, Marburg, Germany. ${ }^{3} \mathrm{Clinic}$ for Gynecology, Gynecological Oncology and Gynecological Endocrinology, University Hospital Giessen and Marburg (UKGM), Marburg, Germany. ${ }^{4}$ Biomolecular Mass Spectrometry, Max-Planck-Institute for Heart and Lung Research, Bad Nauheim, Germany. ${ }^{5}$ The German Centre for Cardiovascular Research (DZHK), Partner Site Rhine-Main, Max Planck Institute for Heart and Lung Research, Bad Nauheim, Germany
}

Conflict of interest

The authors declare that they have no conflict of interest. 


\section{Publisher's note}

Springer Nature remains neutral with regard to jurisdictional claims in published maps and institutional affiliations.

Supplementary Information accompanies this paper at (https://doi.org/ 10.1038/s41419-020-2438-8).

Received: 2 December 2019 Revised: 10 March 2020 Accepted: 10 March 2020

Published online: 20 April 2020

\section{References}

1. Narod, S. Can advanced-stage ovarian cancer be cured? Nat. Rev. Clin. Oncol. 13, 255-261 (2016).

2. Pogge von Strandmann, E., Reinartz, S., Wager, U. \& Müller, R. Tumor-host cell interactions in ovarian cancer: pathways to therapy failure. Trends Cancer $\mathbf{3}$, 137-148 (2017).

3. Lengyel, E. Ovarian cancer development and metastasis. Am. J. Pathol. 177, 1053-1064 (2010)

4. Pradeep, $\mathrm{S}$. et al. Hematogenous metastasis of ovarian cancer: rethinking mode of spread. Cancer Cell 26, 77-91 (2014).

5. Sehouli, J. et al. Intra-abdominal tumor dissemination pattern and surgical outcome in 214 patients with primary ovarian cancer. J. Surg. Oncol. 99, 424-427 (2009).

6. Latifi, A. et al. Isolation and characterization of tumor cells from the ascites of ovarian cancer patients: molecular phenotype of chemoresistant ovarian tumors. PLOS ONE 7, e46858 (2012).

7. Worzfeld, T. et al. Proteotranscriptomics reveal signaling networks in the ovarian cancer microenvironment. Mol. Cell. Proteomics 17, 270-289 (2018).

8. Reinartz, S. et al. Mixed-polarization phenotype of ascites-associated macrophages in human ovarian carcinoma: Correlation of CD163 expression, cytokine levels and early relapse. Int. J. Cancer 134, 32-42 (2014).

9. Kulbe, $\mathrm{H}$. et al. A dynamic inflammatory cytokine network in the human ovarian cancer microenvironment. Cancer Res. 72, 66-75 (2012).

10. Peng, P., Yan, Y. \& Keng, S. Exosomes in the ascites of ovarian cancer patients: origin and effects on anti-tumor immunity. Oncol. Rep. 25, 749-762 (2011).

11. Reiners, K. S. et al. Soluble ligands for NK cell receptors promote evasion of chronic lymphocytic leukemia cells from NK cell anti-tumor activity. Blood 121 3658-3665 (2013).

12. Sever, R. \& Brugge, J. S. Signal transduction in cancer. Cold Spring Harb. Perspect. Med. 5, a006098 (2015).

13. Finkernagel, F. et al. The transcriptional signature of human ovarian carcinoma macrophages is associated with extracellular matrix reorganization. Oncotarget 7, 75339-75352 (2016)

14. Condeelis, J. \& Pollard, J. W. Macrophages: obligate partners for tumor cell migration, invasion, and metastasis. Cell 124, 263-266 (2006).

15. Sica, A. \& Bronte, V. Altered macrophage differentiation and immune dysfunction in tumor development. J. Clin. Invest. 117, 1155-1166 (2007).

16. Qian, B.-Z. \& Pollard, J. W. Macrophage diversity enhances tumor progression and metastasis. Cell 141, 39-51 (2010).

17. Adhikary, T. et al. Interferon signaling in ascites-associated macrophages is linked to a favorable clinical outcome in a subgroup of ovarian carcinoma patients. BMC Genomics 18, 1053 (2017).

18. Quatromoni, J. G. \& Eruslanov, E. Tumor-associated macrophages: function, phenotype, and link to prognosis in human lung cancer. Am. J. Transl. Res. 4, 376-389 (2012)

19. Rodriguez, G. C. et al. Regulation of invasion of epithelial ovarian cancer by transforming growth factor- $\beta$. Gynecol. Oncol. 80, 245-253 (2001).

20. Worzfeld, T. et al. The unique molecular and cellular microenvironment of ovarian cancer. Front. Oncol. 7, v23 (2017).

21. Lewis, C. E. \& Pollard, J. W. Distinct role of macrophages in different tumor microenvironments. Cancer Res. 66, 605-612 (2006).

22. Liu, L. et al. Upregulation of IGF1 by tumor-associated macrophages promotes the proliferation and migration of epithelial ovarian cancer cells. Oncol. Rep. 39, 818-826 (2018).

23. Zeng, X.-Y. et al. M2-like tumor-associated macrophages-secreted EGF promotes epithelial ovarian cancer metastasis via activating EGFR-ERK signaling and suppressing IncRNA LIMT expression. Cancer Biol. Ther. 20, 956-966 (2019).
24. Chen, Y., Zhang, S., Wang, Q. \& Zhang, X. Tumor-recruited M2 macrophages promote gastric and breast cancer metastasis via M2 macrophage-secreted CHI3L1 protein. J. Hematol. Oncol. 10, 78 (2017).

25. Mantovani, A. et al. The chemokine system in diverse forms of macrophage activation and polarization. Trends Immunol. 25, 677-686 (2004).

26. Kalluri, R. \& Zeisberg, M. Fibroblasts in cancer. Nat. Rev. Cancer 6, 392-401 (2006).

27. Liguori, M., Solinas, G., Germano, G., Mantovani, A. \& Allavena, P. Tumorassociated macrophages as incessant builders and destroyers of the cancer stroma. Cancers 3, 3740-3761 (2011).

28. Paron, I. et al. Tenascin-C enhances pancreatic cancer cell growth and motility and affects cell adhesion through activation of the integrin pathway. PLOS ONE 6, e21684 (2011).

29. Ween, M. P. et al. Transforming growth factor-beta-induced protein secreted by peritoneal cells increases the metastatic potential of ovarian cancer cells. Int. J. Cancer 128, 1570-1584 (2011).

30. Yousif, N. G. Fibronectin promotes migration and invasion of ovarian cancer cells through up-regulation of FAK-PI3K/Akt pathway. Cell Biol. Int. 38, 85-91 (2014).

31. Finkernagel, F. et al. Dual-platform affinity proteomics identifies links between the recurrence of ovarian carcinoma and proteins released into the tumor microenvironment. Theranostics 9, 6601-6617 (2019).

32. Lollo, B., Steele, F. \& Gold, L. Beyond antibodies: new affinity reagents to unlock the proteome. Proteomics 14, 638-644 (2014).

33. Reinartz, S. et al. A transcriptome-based global map of signaling pathways in the ovarian cancer microenvironment associated with clinical outcome. Genome Biol. 17, 1053 (2016).

34. The Cancer Genome Atlas Research Network. Integrated genomic analyses of ovarian carcinoma. Nature 474, 609-615 (2011).

35. Gyorffy, B., Lanczky, A. \& Szallasi, Z. Implementing an online tool for genome-wide validation of survival-associated biomarkers in ovariancancer using microarray data from 1287 patients. Endocr. Relat. Cancer 19, 197-208 (2012).

36. Gentles, A. J. et al. The prognostic landscape of genes and infiltrating immune cells across human cancers. Nat. Med. 21, 938-945 (2015).

37. Thapa, N., Lee, B.-H. \& Kim, I.-S. TGFBlp/ßig-h3 protein: a versatile matrix molecule induced by TGF-B. Int. J. Biochem. Cell Biol. 39, 2183-2194 (2007).

38. Tumbarello, D. A., Andrews, M. R., Brenton, J. D. \& Cukierman, E. SPARC regulates transforming growth factor beta induced (TGFBI) extracellular matrix deposition and paclitaxel response in ovarian cancer cells. PLOS ONE 11, e0162698 (2016).

39. Swindle, C. S. et al. Epidermal growth factor (EGF)-like repeats of human tenascin-C as ligands for EGF receptor. J. Cell Biol. 154, 459-468 (2001).

40. Zhu, J., Chen, X., Liao, Z., He, C. \& Hu, X. TGFBI protein high expression predicts poor prognosis in colorectal cancer patients. Int. J. Clin. Exp. Pathol. 8, 702-710 (2015).

41. Ozawa, D. et al. TGFBI expression in cancer stromal cells is associated with poor prognosis and hematogenous recurrence in esophageal squamous cell carcinoma. Ann. Surg. Oncol. 23, 282-289 (2016).

42. Gocheva, V. et al. Quantitative proteomics identify Tenascin-C as a promoter of lung cancer progression and contributor to a signature prognostic of patient survival. Proc. Natl Acad. Sci. USA 114, E5625 (2017).

43. Yang, Z. et al. Tenascin-C as a prognostic determinant of colorectal cancer through induction of epithelial-to-mesenchymal transition and proliferation. Exp. Mol. Pathol. 105, 216-222 (2018).

44. Ma, L.-J. et al. Fibronectin overexpression is associated with latent membrane protein 1 expression and has independent prognostic value for nasopharyngeal carcinoma. Tumor Biol. 35, 1703-1712 (2014).

45. Gopal, S. et al. Fibronectin-guided migration of carcinoma collectives. Nat. Commun. 8, 14105 (2017).

46. Billings, $P$. C. et al. The transforming growth factor- $\beta$-inducible matrix protein Big-h3 interacts with fibronectin. J. Biol. Chem. 277, 28003-28009 (2002).

47. Hanssen, E., Reinboth, B. \& Gibson, M. A. Covalent and non-covalent interactions of Big-h3 with collagen VI. J. Biol. Chem. 278, 24334-24341 (2003).

48. Kim, J.-E. et al. Identification of motifs for cell adhesion within the repeated domains of transforming growth factor- $\beta$-induced gene, Big-h3. J. Biol. Chem 275, 30907-30915 (2000).

49. Son, H.-N., Nam, J.-O., Kim, S. \& Kim, I.-S. Multiple FAS1 domains and the RGD motif of TGFBI act cooperatively to bind av 33 integrin, leading to antiangiogenic and anti-tumor effects. Biochim. Biophys. Acta Mol. Cell Res. 1833, 2378-2388 (2013). 
50. LeBaron, R. G. et al. $\beta \mid G-H 3$, a novel secretory protein inducible by transforming growth factor- $\beta$, is present in normal skin and promotes the adhesion and spreading of dermal fibroblasts in vitro. J. Invest. Dermatol. 104, 844-849 (1995).

51. Gratchev, A. et al. Alternatively activated macrophages differentially express fibronectin and its splice variants and the extracellular matrix protein betalGH3. Scand. J. Immunol. 53, 386-392 (2001).

52. Nam, J.-O. et al. Identification of the $a \vee \beta 3$ integrin-interacting motif of $\beta$ ig-h3 and its anti-angiogenic effect. J. Biol. Chem. 278, 25902-25909 (2003).

53. Suzuki, M. et al. High stromal transforming growth factor $\beta$-induced expression is a novel marker of progression and poor prognosis in gastric cancer. J. Surg. Oncol. 118, 966-974 (2018).

54. Ween, M. P., Oehler, M. K. \& Ricciardelli, C. Transforming growth factor-betainduced protein (TGFBI)/(Big-H3): a matrix protein with dual functions in ovarian cancer. Int. J. Mol. Sci 13, 10461-10477 (2012).

55. Nummela, P. et al. Transforming growth factor beta-induced (TGFBI) is an antiadhesive protein regulating the invasive growth of melanoma cells. Am. J. Pathol. 180, 1663-1674 (2012).

56. Lauden, L. et al. TGF- $\beta$-induced (TGFBI) protein in melanoma: a signature of high metastatic potential. J. Investigative Dermatol. 134, 1675-1685 (2014).

57. Shang, D., Liu, Y., Yang, P., Chen, Y. \& Tian, Y. TGFBl-promoted adhesion, migration and invasion of human renal cell carcinoma depends on inactivation of von Hippel-Lindau tumor suppressor. Urology 79, $966 . e 1$ (2012).

58. Midwood, K. S., Chiquet, M., Tucker, R. P. \& Orend, G. Tenascin-C at a glance. J. Cell Sci. 129, 4321-4327 (2016).

59. Giblin, S. P. \& Midwood, K. S. Tenascin-C: form versus function. Cell Adh. Migr. 9, 48-82 (2015).

60. Midwood, K. S., Hussenet, T., Langlois, B. \& Orend, G. Advances in tenascin-C biology. Cell. Mol. Life Sci. 68, 3175-3199 (2011).

61. Lowy, C. M. \& Oskarsson, T. Tenascin C in metastasis: a view from the invasive front. Cell Adh. Migr. 9, 112-124 (2015).

62. Boeck, A. et al. Differential secretome analysis of cancer-associated fibroblasts and bone marrow-derived precursors to identify microenvironmental regulators of colon cancer progression. Proteomics 13, 379-388 (2013).

63. Santi, A., Kugeratski, F. G. \& Zanivan, S. Cancer associated fibroblasts: the architects of stroma remodeling. Proteomics 18, 1700167 (2018).

64. Wallner, K. et al. Tenascin-C is expressed in macrophage-rich human coronary atherosclerotic plaque. Circulation 99, 1284-1289 (1999).

65. Borsi, L. et al. Expression of different tenascin isoforms in normal, hyperplastic and neoplastic human breast tissues. Int. J. Cancer 52, 688-692 (1992).
66. Grahovac, J. \& Wells, A. Matrikine and matricellular regulators of EGF receptor signaling on cancer cell migration and invasion. Lab. Invest. 94, 31-40 (2014).

67. Tucker, R. P. \& Chiquet-Ehrismann, R. Tenascin-C: its functions as an integrin ligand. Int. J. Biochem. Cell Biol. 65, 165-168 (2015).

68. Yoshida, T., Akatsuka, T. \& Imanaka-Yoshida, K. Tenascin-C and integrins in cancer. Cell Adh. Migr. 9, 96-104 (2015).

69. Orend, G. \& Chiquet-Ehrismann, R. Tenascin-C induced signaling in cancer. Cancer Lett. 244, 143-163 (2006).

70. Ince, T. A. et al. Characterization of twenty-five ovarian tumour cell lines that phenocopy primary tumours. Nat. Commun. 6, 264 (2015).

71. Reinartz, S. et al. Cell type-selective pathways and clinical associations of lysophosphatidic acid biosynthesis and signaling in the ovarian cancer microenvironment. Mol. Oncol. 13, 185-201 (2019).

72. Rappsilber, J., Ishihama, Y. \& Mann, M. Stop and go extraction tips for matrix-assisted laser desorption/ionization, nanoelectrospray, and LC/ MS sample pretreatment in proteomics. Anal. Chem. 75, 663-670 (2003).

73. Perez-Riverol, Y. et al. The PRIDE database and related tools and resources in 2019: improving support for quantification data. Nucleic Acids Res. 47, D442 (2019).

74. Kiweler, M., Looso, M. \& Graumann, J. MARMoSET - extracting publicationready mass spectrometry metadata from RAW files. Mol. Cell. Proteomics 18 1700-1702 (2019)

75. Cox, J. et al. Accurate proteome-wide label-free quantification by delayed normalization and maximal peptide ratio extraction, termed MaxLFQ. Mol. Cell. Proteomics 13, 2513-2526 (2014).

76. Cox, J. \& Mann, M. MaxQuant enables high peptide identification rates, individualized p.p.b.-range mass accuracies and proteome-wide protein quantification. Nat. Biotechnol. 26, 1367-1372 (2008).

77. Cox, J. et al. Andromeda: a peptide search engine integrated into the MaxQuant environment. J. Proteome Res. 10, 1794-1805 (2011).

78. Reinartz, S., Failer, S., Schuell, T. \& Wagner, U. CA125 (MUC16) gene silencing suppresses growth properties of ovarian and breast cancer cells. Eur. J. Cancer 48, 1558-1569 (2012)

79. Unger, A et al. Chromatin binding of c-REL and p65 is not limiting for macrophage IL12B transcription during immediate suppression by ovarian carcinoma ascites. Front. Immunol. 9, 254 (2018).

80. Guescini, M., Sisti, D., Rocchi, M. B. L., Stocchi, L. \& Stocchi, V. A new real-time PCR method to overcome significant quantitative inaccuracy due to slight amplification inhibition. BMC Bioinformatics 9, 1026 (2008). 